Pacific

Journal of

Mathematics

BOUNDARIES OF HYPERBOLIC METRIC SPACES

CORRAN WEBStER AND ADAM Winchester 


\title{
BOUNDARIES OF HYPERBOLIC METRIC SPACES
}

\author{
CORRAN WEBSTER AND ADAM Winchester
}

\begin{abstract}
We investigate the relationship between the metric boundary and the Gromov boundary of a hyperbolic metric space. We show that the Gromov boundary is a quotient of the metric boundary and the quotient map is continuous, and that therefore a word-hyperbolic group has an amenable action on the metric boundary of its Cayley graph. Furthermore, if the space is 0-hyperbolic, the boundaries agree, and as a consequence there are no non-Busemann points on the boundary of such spaces. These results have significance for the study of Lip-norms on group $C^{*}$-algebras.
\end{abstract}

\section{Introduction}

The Gromov boundary of a hyperbolic metric space has been extensively studied, but the Gromov boundary is not guaranteed to exist for nonhyperbolic metric spaces. Gromov [1981] introduced another boundary which makes sense for any metric space, but this was little studied until Marc Rieffel [2002] showed that this second boundary, called the metric boundary in his papers, is important in the study of metrics on the state spaces of group $C^{*}$-algebras.

If $G$ is a countable discrete group equipped with a length function $\ell$, and $C_{r}^{*}(G)$ is its reduced $C^{*}$-algebra, there is a seminorm $L_{\ell}(f)=\left\|\left[M_{\ell}, f\right]\right\|$ defined on a dense *-subalgebra of $C_{r}^{*}(G)$, where $M_{\ell}$ is multiplication by $\ell$ and $f$ operates by convolution on $\ell^{2}(G)$. This in turn gives a metric on the state space of $C_{r}^{*}(G)$ by

$$
\rho_{L_{\ell}}(\varphi, \psi)=\sup \left\{|\varphi(f)-\psi(f)|: L_{\ell}(f) \leq 1\right\},
$$

and a natural question to ask is whether the topology generated by this metric coincides with the weak-* topology on the state space, that is, whether the seminorm is a Lip-norm [Rieffel 1998; 1999; 2004]. Rieffel proves that this is in fact the case for $\mathbb{Z}^{d}$ with certain length functions, and a critical requirement in his proof is that the action of $\mathbb{Z}^{d}$ on its metric boundary is always amenable.

There is some interest, then, in knowing when the action of a group is amenable on its metric boundary. In the case of word-hyperbolic groups with the standard

MSC2000: primary 20F65; secondary 46L87, 53C23.

Keywords: hyperbolic space, hyperbolic group, Gromov boundary, Cayley graph, group

$C *$-algebra, quantum metric space. 
word-length metric, it is known that the action of a word-hyperbolic group on its Gromov boundary is amenable [Anantharaman-Delaroche and Renault 2000; Anantharaman-Delaroche 2002], and as Rieffel points out [2002], if there is an equivariant, continuous surjection from the metric boundary onto the Gromov boundary, then the action of the group on the metric boundary must be amenable.

We show that this is in fact the case, and more: for 0-hyperbolic spaces, the Gromov boundary and the metric boundary are in fact identical, with identical topologies.

We note here that Ozawa and Rieffel [2003] have shown that, for hyperbolic groups, $L_{\ell}$ is in fact a Lip-norm using techniques which do not use the notion of the metric boundary. However these methods do not work for $\mathbb{Z}^{d}$, and we hope that our result may be part of a unified way of showing that the seminorms for these groups are in fact Lip-norms.

\section{The Gromov boundary}

There are many different but equivalent definitions for a hyperbolic metric space, but for our purposes we are only interested in a couple. We follow [Alonso et al. 1991] in our presentation, and a more complete discussion of hyperbolic spaces can be found there.

Definition 2.1. A metric space $(X, d)$ is geodesic if given any two points $x, y \in X$, there is an isometry $\gamma$ from the interval $[0, d(x, y)]$ into $X$.

If $(X, d)$ is a metric space, with a base-point 0 , we define an inner product by

$$
(x \cdot y)_{0}=\frac{1}{2}(d(x, 0)+d(y, 0)-d(x, y)) .
$$

Where the base point is implicit, we will just write $(x \cdot y)$.

The metric space $(X, d)$ is hyperbolic if it is geodesic and there is some $\delta \geq 0$ such that

$$
(x \cdot y) \geq \min \{(x \cdot z),(y \cdot z)\}-\delta
$$

for all $z \in X$.

One can show that although the constant $\delta$ may be different for different basepoints, whether the space is hyperbolic does not depend on the choice of base-point.

It is worth noting that not all references do not require hyperbolic spaces to be geodesic; however we need the space to be geodesic at a key place (Lemma 4.1).

We have a particular interest in groups whose Cayley graphs are hyperbolic, and there is an equivalent definition based on properties of generators and relations alone. We note that if $G$ is a group with a finite presentation $\langle S \mid R\rangle$, then given a 
reduced word $w$ in the generators, $S$, with $w=e$ in $G$, we can write $w$ as a product

$$
w=\prod_{k=1}^{n} u_{k}^{-1} r_{k} u_{k},
$$

where $u_{k}$ is a word in $S \cup S^{-1}$ and $r_{k} \in R \cup R^{-1}$. For a given $w$, let $n_{w}$ be the smallest possible number of terms in such a product, and let $l(w)$ be the length of $w$.

Definition 2.2. Let $G$ be a group with a finite presentation $\langle S \mid R\rangle$. We say that $G$ is word-hyperbolic if it satisfies a linear isoperimetric inequality: there is some $K \geq 0$ such that $n_{w} \leq K l(w)$ for all reduced words $w$ with $w=e$ in $G$.

One can show that the choice of generators and relations does not affect whether the group is word-hyperbolic and, moreover, a group is word-hyperbolic if and only if its Cayley graph (regarded as a 1-complex with the graph metric) is hyperbolic.

Perhaps the simplest way to consider the Gromov boundary is as the limit points of geodesic rays, where two geodesic rays are considered equivalent if they are a finite distance apart. This definition highlights similarities between the Gromov boundary and the metric boundary discussed in the next section. However, the most useful definition of the Gromov boundary for our purposes is in terms of the inner product.

Definition 2.3. Let $(X, d)$ be a metric space. We say that a sequence $x_{k}$ converges to infinity (in the Gromov sense) if

$$
\lim _{n, k \rightarrow \infty}\left(x_{n} \cdot x_{k}\right)=\infty
$$

Given two sequences $x=\left(x_{n}\right)_{n=1}^{\infty}$ and $y=\left(y_{n}\right)_{n=1}^{\infty}$ which both converge to infinity, we define a relation $\sim_{G}$ by

$$
x \sim_{G} y \Longleftrightarrow \lim _{n \rightarrow \infty}\left(x_{n} \cdot y_{n}\right)=\infty .
$$

If $(X, d)$ is a hyperbolic metric space, then $\sim_{G}$ is in fact an equivalence relation on sequences which converge to infinity. It is worthwhile noting that if $(X, d)$ is hyperbolic then

$$
x \sim_{G} y \Longleftrightarrow \lim _{n, k \rightarrow \infty}\left(x_{n} \cdot y_{k}\right)=\infty .
$$

If $(X, d)$ is not hyperbolic, the relation $\sim_{G}$ will not, in general, be an equivalence relation.

We define the Gromov boundary $\partial_{G} X$ of a hyperbolic metric space $(X, d)$ to be the set of equivalence classes of sequences which converge to infinity. We will say that a sequence in $X$ converges to an equivalence class in $\partial_{G} X$ if it is an element of the equivalence class. 
We can topologize the boundary by extending the inner product to

$$
\bar{X}^{G}=X \cup \partial_{G} X .
$$

Definition 2.4. Let $(X, d)$ be a hyperbolic metric space, and let $x, y \in \bar{X}^{G}$. Then we define

$$
(x \cdot y)=\inf \left\{\liminf _{n}\left(x_{n} \cdot y_{n}\right): x_{n} \rightarrow x, y_{n} \rightarrow y, \text { and } x_{n}, y_{n} \in X\right\} .
$$

One can show that if this inner product is restricted to $X$, it is the same as the original inner product on $X$. Furthermore, if $\omega \in \partial_{G} X$, and $y \in X$, we have

$$
(\omega \cdot y)=\inf \left\{\liminf _{n}\left(x_{n} \cdot y\right): x_{n} \rightarrow \omega, \text { and } x_{n} \in X\right\} .
$$

It is also the case that if $(X, d)$ is hyperbolic, with

$$
(x \cdot y) \geq \min \{(x \cdot z),(y \cdot z)\}-\delta
$$

for all $x, y$ and $z \in X$, then the same identity holds for this extended inner product. We have

$$
(x \cdot y) \geq \min \{(x \cdot z),(y \cdot z)\}-\delta,
$$

for all $x, y$ and $z \in \bar{X}^{G}$.

We then can say that a sequence $x_{n} \in \bar{X}^{G}$ converges to $\omega \in \partial_{G} X$ if and only if

$$
\left(\omega \cdot x_{n}\right) \rightarrow \infty
$$

With this definition, it can be shown that $\bar{X}^{G}$ is a compactification of $X$ provided $X$ is locally compact.

\section{The metric boundary}

We now consider the metric compactification and the metric boundary. The most succinct definition is that the metric compactification $\bar{X}^{d}$ of a locally compact metric space $(X, d)$ corresponds to the pure states of the commutative, unital, $C^{*}$-algebra $\mathscr{G}(X, d)$ generated by the functions which vanish at infinity on $X$, the constant functions, and the functions of the form

$$
\varphi_{y}(x)=d(x, 0)-d(x, y),
$$

where 0 is some fixed base-point (which does not affect the resulting algebra). The metric boundary $\partial_{d} X$ is simply $\bar{X}^{d} \backslash X$.

More concretely, we can understand the metric boundary as a limit of rays in much the same way as the simple definition of the Gromov boundary.

Definition 3.1. Let $(X, d)$ be a metric space, and $T$ an unbounded subset of $\mathbb{R}^{+}$ containing 0 , and let $\gamma: T \rightarrow X$. 
(1) $\gamma$ is a geodesic ray if

$$
d(\gamma(s), \gamma(t))=|s-t|
$$

for all $s, t \in T$.

(2) $\gamma$ is an almost geodesic ray if for every $\varepsilon>0$, there is an integer $N$ such that

$$
|d(\gamma(t), \gamma(s))+d(\gamma(s), \gamma(0))-t|<\varepsilon
$$

for all $t, s \in T$ with $t \geq s \geq N$.

(3) $\gamma$ is a weakly geodesic ray if for every $y \in X$ and every $\varepsilon>0$, there is an integer $N$ such that

$$
|d(\gamma(t), \gamma(0))-t|<\varepsilon
$$

and

$$
|d(\gamma(t), y)-d(\gamma(s), y)-(t-s)|<\varepsilon
$$

for all $t, s \in T$ with $t, s \geq N$.

It is immediate that every geodesic ray is an almost geodesic ray. Rieffel showed that every almost geodesic ray is a weakly geodesic ray. The significance of weakly geodesic rays is that they give the points on the metric boundary in reasonable metric spaces.

Theorem 3.2 (Rieffel). Let $(X, d)$ be a complete, locally compact metric space, and let $\gamma: T \rightarrow X$ be a weakly geodesic ray in $X$. Then

$$
\lim _{t \rightarrow \infty} f(\gamma(t))
$$

exists for every $f \in \mathscr{G}(X, d)$, and defines an element of $\partial_{d} X$. Conversely, if $d$ is proper and if $(X, d)$ has a countable base, then every point of $\partial_{d} X$ is determined as above by a weakly geodesic ray.

This is similar in character to the definition of the Gromov boundary, although the reliance on weakly geodesic rays is necessary in general. Rieffel defined any point $\partial_{d} X$ which is the limit of an almost geodesic ray to be a Busemann point, and it was shown in [Webster and Winchester 2003] that even for simple hyperbolic spaces the metric boundary may have non-Busemann points. It is an open question whether this phenomenon can occur with word-hyperbolic groups. We will show later that if the space is 0-hyperbolic then there are no non-Busemann points.

Unlike the Gromov boundary, the metric boundary is, in general, dependent upon the choice of metric. For example, different generating sets for an infinite discrete group generally give distinct metric boundaries for the corresponding wordlength metrics.

From a practical viewpoint, the initial definition of the metric boundary means that a sequence $x_{n} \in X$ converges to a point on the metric boundary if and only 
if $x_{n}$ is eventually outside any compact subset of $X$, and $\varphi_{y}\left(x_{n}\right)$ converges for all $y \in X$. Two sequences converge to the same point on the metric boundary if and only if

$$
\lim _{n \rightarrow \infty} \varphi_{z}\left(x_{n}\right)=\lim _{k \rightarrow \infty} \varphi_{z}\left(y_{k}\right)
$$

for every $z \in X$. We can extend the functions $\varphi_{y}$ to the boundary by letting

$$
\varphi_{y}(\omega)=\lim _{n \rightarrow \infty} \varphi_{y}\left(x_{n}\right)
$$

for any sequence $x_{n} \rightarrow \omega \in \partial_{d} X$. Then a sequence $x_{n} \in \bar{X}^{d}$ converges to $x \in \partial_{d} X$ if and only if $\varphi_{y}\left(x_{n}\right) \rightarrow \varphi_{y}(x)$ for all $y \in X$, and this is sufficient to determine the topology of the metric compactification.

\section{The Gromov boundary as a quotient}

We observe that the functions $\varphi_{y}$ and the inner product are closely related, since

$$
(x \cdot y)=\frac{1}{2}\left(\varphi_{y}(x)+d(y, 0)\right),
$$

and furthermore, that they play similar roles in the definitions of Gromov and metric boundaries. It is natural, therefore, to ask what relationship there may be between the two different boundaries.

The key observation is that the triangle inequality implies that for any $z \in X$,

$$
(x \cdot y) \geq \frac{1}{2}(d(x, 0)+d(y, 0)-d(x, z)-d(y, z))=\frac{1}{2}\left(\varphi_{z}(x)+\varphi_{z}(y)\right),
$$

with equality if and only if $z$ lies on a geodesic path $[x, y]$. We will want to show that $(x \cdot y)$ gets large for elements from various sequences, and this implies that all we need do is find a $z$ so that $\varphi_{z}(x)+\varphi_{z}(y)$ is large.

The following lemma tells us that as we get close to a metric boundary point, we can find $z$ such that $\varphi_{z}$ is large.

Lemma 4.1. Let $(X, d)$ be a proper geodesic metric space with a distinguished base-point 0 . Then for any $\omega$ in the metric boundary of $X$, and any $N$, there is a point $z \in X$ such that $\varphi_{z}(\omega)>N$.

Proof. Let $x_{v}$ be any net which converges to $\omega$.

Let $r>0$ and consider a collection of minimal paths [0, $\left.x_{v}\right]$ for $n$ large enough that $d\left(0, x_{v}\right)>r$. Because $(X, d)$ is a geodesic metric space, there must be a unique point $y_{\nu}$ in each of these paths in the sphere $S(0, r)$ of radius $r$, centered at 0 . Since $(X, d)$ is proper the sphere $S(0, r)$ is compact, and hence $y_{\nu}$ has a convergent subnet $y_{v_{\lambda}}$ which converges to some $z_{r} \in S(0, r)$. Let $x_{v_{\lambda}}$ be the corresponding subnet of $x_{v}$. Given any $\varepsilon>0$ we have for $r>\varepsilon$ and $\lambda$ sufficiently large,

$$
d\left(0, x_{v_{\lambda}}\right)=d\left(0, y_{v_{\lambda}}\right)+d\left(y_{\nu_{\lambda}}, x_{v_{\lambda}}\right)>d\left(0, z_{r}\right)+d\left(z_{r}, x_{v_{\lambda}}\right)-\varepsilon,
$$


or, equivalently,

$$
\varphi_{z_{r}}\left(x_{v_{\lambda}}\right)=d\left(0, x_{v_{\lambda}}\right)-d\left(z_{r}, x_{v_{\lambda}}\right)>d\left(0, z_{r}\right)-\varepsilon=r-\varepsilon .
$$

Taking limits, we conclude that

$$
\varphi_{z_{r}}(\omega) \geq r-\varepsilon .
$$

Hence, given any $N$, we can choose $r$ and $\varepsilon$ such that $r-\varepsilon>N$, and obtain a point $z$ such that $\varphi_{z}(\omega)>N$.

We do not know if there is a counterexample for this result if the requirement that the hyperbolic space be geodesic is dropped. Clearly one does not need the full force of the geodesic requirement to produce an appropriate collection of elements $z_{r}$. For example, it would suffice to have a condition that there is some constant $C$ and some unbounded subset $T \subseteq \mathbb{R}$ such that for every $x \in X$ there is a function

$$
f:\{t \in T: 0 \leq t \leq d(x, 0)\} \rightarrow X
$$

such that

$$
d(0, x)+C \geq d(0, f(t))+d(f(t), x) .
$$

The proof would proceed as above for $r \in T$, and would give the estimate

$$
\varphi_{z_{r}} \geq r-C-\varepsilon .
$$

This condition is satisfied by Cayley graphs of hyperbolic groups, for instance, when regarded as a set of points with the graph metric rather than as a 1-complex.

Although much of what follows may be applicable in more general settings, we will assume that our metric space has a countable base so that we may use sequences instead of nets.

The lemma above has two immediate corollaries:

Corollary 4.2. Let $(X, d)$ be a proper geodesic metric space with a countable base and a distinguished base-point 0 , and let $x_{n} \rightarrow \omega \in \partial_{d} X$. Then $x_{n}$ converges to infinity in the Gromov sense.

Proof. We know that for all $z, \varphi_{z}\left(x_{n}\right)$ eventually gets close to $\varphi_{z}(\omega)$. Hence by the previous lemma, for any $N$ we can find a $z$ such that $\varphi_{z}\left(x_{n}\right)>N$ for all $n$ sufficiently large. It follows that if $x_{n}$ and $x_{m}$ are large enough to make $\varphi_{z}\left(x_{n}\right)$ and $\varphi_{z}\left(x_{n}\right)$ greater than $N$, we have

$$
\left(x_{n} \cdot x_{m}\right) \geq \frac{1}{2}\left(\varphi_{z}\left(x_{n}\right)+\varphi_{z}\left(x_{m}\right)\right)>N \text {. }
$$

Therefore

$$
\lim _{n, m \rightarrow \infty}\left(x_{n} \cdot x_{m}\right)=\infty,
$$

and so $x_{n}$ goes to infinity in the Gromov sense. 
Let $\left(x_{n}\right)$ and $\left(y_{k}\right)$ be two sequences in $X$ which converge to points on the metric boundary. We will say that $\left(x_{n}\right) \sim_{d}\left(y_{k}\right)$ if these two sequences converge to the same metric boundary point.

Corollary 4.3. Let $(X, d)$ be a proper geodesic metric space with a countable base. Then $\left(x_{n}\right) \sim_{d}\left(y_{k}\right)$ implies $\left(x_{n}\right) \sim_{G}\left(y_{k}\right)$.

Proof. Let $x_{n}$ and $y_{n}$ both converge to $\omega$. Using the lemma, we can find a point $z$ so that $\varphi_{z}(\omega)$ is arbitrarily large, and since both $\varphi_{z}\left(x_{n}\right)$ and $\varphi_{z}\left(y_{n}\right)$ converge to $\varphi_{z}(\omega)$, for any number $N$ we can find $z$ such that both $\varphi_{z}\left(x_{n}\right)$ and $\varphi_{z}\left(y_{n}\right)$ are greater then $N$ for all $n$ sufficiently large.

Hence

$$
\left(x_{n} \cdot y_{n}\right) \geq \frac{1}{2}\left(\varphi_{z}\left(x_{n}\right)+\varphi_{z}\left(y_{n}\right)\right)>N
$$

for all $n$ sufficiently large, and so

$$
\lim _{n \rightarrow \infty}\left(x_{n} \cdot y_{n}\right)=\infty
$$

and so $\left(x_{n}\right) \sim_{G}\left(y_{k}\right)$.

Recall from the preliminary discussion that another way of describing the Gromov boundary is as equivalence classes of sequences which stay a finite distance from each other. This is an equivalence relation on sequences, which we may denote by $\sim_{G^{\prime}}$, and for hyperbolic metric spaces it is equal to $\sim_{G}$. It is worth noting, however, that for spaces which are not hyperbolic it is not the case that $\left(x_{n}\right) \sim_{d}\left(y_{k}\right)$ implies $\left(x_{n}\right) \sim_{G^{\prime}}\left(y_{k}\right)$. For example, in $\mathbb{Z}^{2}$ with the graph metric given by the standard generators $(1,0)$ and $(0,1)$, we have that $x_{n}=(n, n)$ and $y_{k}=(k, 2 k)$ both converge to the same point on the metric boundary, but clearly $\left(x_{n}\right) \chi_{G^{\prime}}\left(y_{k}\right)$.

These two corollaries mean that we have a well-defined relation $\sim$ on $\partial_{d} X$ given by $\omega_{1} \sim \omega_{2}$ if and only if, given any $x_{n} \rightarrow \omega_{1}$ and $y_{k} \rightarrow \omega_{2}$, we have $\left(x_{n}\right) \sim_{G}\left(y_{k}\right)$. Furthermore, if $\sim_{G}$ is an equivalence relation (as it is for hyperbolic spaces), then $\sim$ is an equivalence relation on $\partial_{d} X$. As usual, we will denote the equivalence class of a point $\omega$ in the metric boundary by $[\omega]$.

Recalling again the simple definition of the Gromov boundary in terms of equivalence classes of geodesic rays, and the fact that every geodesic ray converges to a point on the metric boundary, we conclude that the quotient map $\pi_{\sim}: \partial_{d} X \rightarrow \partial_{G} X$ is surjective.

In fact we can specify the equivalence relation $\sim_{G}$ directly in terms of the metric boundary.

Proposition 4.4. If $(X, d)$ is a complete, proper $\delta$-hyperbolic metric space with a countable base then given $\omega_{1}$ and $\omega_{2} \in \partial_{d} X$, we have $\omega_{1} \sim_{G} \omega_{2}$ if and only if there 
is an $N$ such that

$$
\left|\varphi_{z}\left(\omega_{1}\right)-\varphi_{z}\left(\omega_{2}\right)\right|<N \quad \text { for all } z \in X .
$$

Proof. We first note that if $\omega_{1}$ and $\omega_{2} \in \partial_{d} X$ and there is an $N>0$ such that $\left|\varphi_{z}\left(\omega_{1}\right)-\varphi_{z}\left(\omega_{2}\right)\right|<N$ then it follows immediately that for any $M$, there exists $z \in X$ such that

$$
\varphi_{z}\left(x_{n}\right)>M+N
$$

for all $n$ sufficiently large, and hence

$$
\varphi_{z}\left(y_{n}\right)>M
$$

for all $n$ sufficiently large, and hence $\left(x_{n}, y_{n}\right)>N$ for all $n$ sufficiently large. Therefore $x_{n}$ and $y_{n}$ converge to the same point in the Gromov boundary, and hence $\omega_{1} \sim_{g} \omega_{2}$.

Conversely, if $x_{n} \rightarrow \omega_{1}$ and $y_{n} \rightarrow \omega_{2}$ and $\omega_{1} \sim_{G} \omega_{2}$, then for any $z \in X$,

$$
\varphi_{z}\left(x_{n}\right)=2\left(x_{n} \cdot z\right)-d(z, 0) \geq 2 \min \left\{\left(x_{n} \cdot y_{n}\right),\left(y_{n} \cdot z\right)\right\}-2 \delta-d(z, 0),
$$

and since $\left(x_{n} \cdot y_{n}\right)$ is eventually arbitrarily large, we have that $\varphi_{z}\left(x_{n}\right) \geq \varphi_{z}\left(y_{n}\right)-2 \delta$. The same argument applies with $x_{n}$ and $y_{n}$ reversed, and so we have that, for $n$ sufficiently large, $\varphi_{z}\left(x_{n}\right)$ and $\varphi_{z}\left(y_{n}\right)$ differ by no more than $2 \delta$. Hence, taking limits,

$$
\left|\varphi_{z}\left(\omega_{1}\right)-\varphi_{z}\left(\omega_{2}\right)\right| \leq 2 \delta
$$

for all $z \in X$.

Note that the proof further implies that if such $N$ exists, then $N \leq 2 \delta$. In particular, this implies that if we are in a 0-hyperbolic space, then $\omega_{1} \sim_{G} \omega_{2}$ if and only if $\omega_{1} \sim_{d} \omega_{2}$.

Corollary 4.5. If $(X, d)$ is a complete, proper, 0-hyperbolic metric space with a countable base, then $\partial_{d} X$ has no non-Busemann points.

Proof. Every element of $\partial_{G} X$ is a limit of a geodesic ray, and since $\partial_{d} X=\partial_{G} X$ for 0-hyperbolic metric spaces, and geodesics converge to the metric boundary, we have that every element of $\partial_{d} X$ is the limit of a geodesic ray, and hence a Busemann point.

We now show that the quotient map from $\partial_{d} X$ to $\partial_{G} X$ is continuous.

Proposition 4.6. Let $(X, d)$ be a proper hyperbolic metric space with a countable base. If $\omega_{n} \rightarrow \omega$ in $\partial_{d} X$, then $\left[\omega_{n}\right] \rightarrow[\omega]$ in $\partial_{G} X$.

Proof. Let $\delta>0$ be the hyperbolic constant from (1). We know that we can find $z$ such that $\varphi_{z}(\omega)$ is arbitrarily large, and since we have $\varphi_{z}\left(\omega_{n}\right) \rightarrow \varphi_{z}(\omega)$, we can choose $z$ such that $\varphi_{z}\left(\omega_{n}\right)$ is also arbitrarily large, for all $n$ sufficiently large. 
Indeed, as in the previous corollaries, given any $N>0$ we can find a number $M$ such that for any sequences $x_{k} \rightarrow \omega$ and $x_{n, k} \rightarrow \omega_{n}$, and for all $n>M$, there is a number $K_{n}$ such that

$$
\left(x_{n, k} \cdot x_{k}\right) \geq \frac{1}{2}\left(\varphi_{z}\left(x_{n, k}\right)+\varphi_{z}\left(x_{k}\right)\right)>N+2 \delta \quad \text { for all } k>K_{n} .
$$

Now if $y_{n, k} \rightarrow\left[\omega_{n}\right]$ and $y_{n} \rightarrow[\omega]$, we know that we can find a subsequence of each sequence such that

$$
\liminf _{k \rightarrow \infty}\left(y_{n, k} \cdot y_{k}\right)=\lim _{k \rightarrow \infty}\left(y_{n, k_{j}} \cdot y_{k_{j}}\right) .
$$

Furthermore, since $x_{n, j} \rightarrow\left[\omega_{n}\right]$ we conclude that for any $N$ there is some $J_{n}$ such that $\left(y_{n, k_{j}} \cdot x_{n, j}\right)>N+2 \delta$ for all $j>J_{n}$, and similarly that there is some $J$ such that $\left(y_{k_{j}} \cdot x_{j}\right)>N+\delta$ for all $j>J$.

Hence, given any $N$, and fixing some $n>M$, we have

$$
\left(y_{n, k_{j}} \cdot x_{j}\right) \geq \min \left\{\left(y_{n, k_{j}} \cdot x_{n, j}\right),\left(x_{n, j} \cdot x_{j}\right)\right\}-\delta>N+\delta
$$

for all $j>\max \left\{J_{n}, K_{n}\right\}$. But then

$$
\left(y_{n, k_{j}} \cdot y_{k_{j}}\right) \geq \min \left\{\left(y_{n, k_{j}} \cdot x_{j}\right),\left(y_{k_{j}} \cdot x_{j}\right)\right\}-\delta>N
$$

for all $j>\max \left\{J_{n}, K_{n}, J\right\}$. Thus $\lim _{k \rightarrow \infty}\left(y_{n, k_{j}} \cdot y_{k_{j}}\right)>N$ for any $n>M$, and so

$$
\liminf _{k \rightarrow \infty}\left(y_{n, k} \cdot y_{k}\right)>N \text { for all } n>M .
$$

And since $M$ does not depend on the choice of sequences converging to $\left[\omega_{n}\right]$ and $[\omega]$, we have

$\left(\left[\omega_{n}\right] \cdot[\omega]\right)=\inf \left\{\liminf _{k \rightarrow \infty}\left(y_{n, k} \cdot y_{k}\right): y_{n, k} \rightarrow\left[\omega_{n}\right], y_{n} \rightarrow[\omega]\right\}>N \quad$ for all $n>M$.

Therefore $\lim _{n \rightarrow \infty}\left(\left[\omega_{n}\right] \cdot[\omega]\right)=\infty$, and so $\left[\omega_{n}\right] \rightarrow[\omega]$ in $\partial_{G} X$.

For 0-hyperbolic spaces, this map is a homeomorphism.

Theorem 4.7. Let $(X, d)$ be a proper, 0-hyperbolic metric space with a countable base. Then $\partial_{d} X$ and $\partial_{G} X$ are homeomorphic.

Proof. If $\omega_{n} \rightarrow \omega$ in $\partial_{G} X$, then given any subsequence of this sequence, we have that it in turn has a subsequence which converges in $\partial_{d} X$ by the compactness of that space, and the limit must be $\omega$. Hence the original sequence converges to $\omega$ in the topology of $\partial_{d} X$. Hence the identity map $\partial_{G} X$ to $\partial_{d} X$ is continuous, and so accounting for the previous proposition, the spaces are homeomorphic.

It is unclear whether the topology on $\partial_{G} X$ is the quotient topology in general. For some cases, such as the example in [Webster and Winchester 2003] which is not 0-hyperbolic, it can be affirmed directly that the map is a quotient map. The authors do not know of any counterexamples. 


\section{Boundaries of word-hyperbolic groups}

We observe that if $G$ is a hyperbolic group, then the group acts on either boundary by taking a sequence $x_{k} \rightarrow \omega$ and letting

$$
\alpha_{g}(\omega)=\lim _{k \rightarrow \infty} g x_{k} .
$$

This is a continuous action on either boundary. Clearly the quotient map is equivariant for these two actions, since if $\omega \sim \omega^{\prime}$, we can easily see that $\alpha_{g}(\omega) \sim \alpha_{g}(\omega)$ by simply changing the base point of the inner product to $g$.

An action of a topological group $G$ on a topological space $X$ is amenable if there is a net of continuous maps

$$
\left(m_{\lambda}: X \rightarrow M_{1}^{+}(G)\right)_{\lambda \in \Lambda},
$$

where $M_{1}^{+}(G)$ is the set of Borel probability measures on $G$, such that

$$
\lim _{\lambda \in \Lambda}\left\|g \cdot m_{\lambda}(x)-m_{\lambda}(g \cdot x)\right\| \rightarrow 0
$$

uniformly on compact subsets of $G \times X$. Such a net of maps is called an approximate invariant continuous mean. It was shown by E. Germain (as discussed in [Anantharaman-Delaroche and Renault 2000; Anantharaman-Delaroche 2002]) that the action of a word-hyperbolic group $G$ on its Gromov boundary is amenable. Rieffel pointed out that if there were a continuous, equivariant surjection from $\partial_{d} G$ to the Gromov boundary, then the action of $G$ on the metric boundary must also be amenable. This is trivial given the above definition, since if $q: \partial_{d} G \rightarrow \partial_{G} G$ is the quotient map of the previous section, and $m_{\lambda}$ are the maps in an approximate invariant continuous mean for the action of $G$ on $\partial_{G} G$, then $m_{\lambda} \circ q$ are an approximate invariant continuous mean for the action of $G$ on $\partial_{d} G$.

Corollary 5.1. If $G$ is word-hyperbolic group with a finite generating set, and $d$ is the word-length metric, then the group action on the metric boundary is amenable.

This would seem to open the possibility of replicating Rieffel's work on the metric boundary of $\mathbb{Z}^{d}$ in the setting of hyperbolic groups. However, Rieffel's procedure relied on the fact that the action of $\mathbb{Z}^{d}$ on its metric boundary always has finite orbits, and it seems unlikely that this criterion holds with any frequency for general hyperbolic groups.

\section{Acknowledgements}

This paper is part of an undergraduate research project between the authors. The authors thank Michelle Schultz for organizing the undergraduate research seminar at UNLV, and Marc Rieffel for encouraging this line of research. 


\section{References}

[Alonso et al. 1991] J. M. Alonso et al., "Notes on word hyperbolic groups", pp. 3-63 in Group theory from a geometrical viewpoint (Trieste, 1990), edited by E. Ghys et al., World Sci. Publishing, River Edge, NJ, 1991. MR 93g:57001 Zbl 0849.20023

[Anantharaman-Delaroche 2002] C. Anantharaman-Delaroche, "Amenability and exactness for dynamical systems and their $C^{*}$-algebras", Trans. Amer. Math. Society 354:10 (2002), 4153-4178. MR 2004e:46082 Zbl 1035.46039

[Anantharaman-Delaroche and Renault 2000] C. Anantharaman-Delaroche and J. Renault, Amenable groupoids, Monographies de L'Enseignement Mathématique 36, L'Enseignement Mathématique, Geneva, 2000. MR 2001m:22005 Zbl 0960.43003

[Gromov 1981] M. Gromov, "Hyperbolic manifolds, groups and actions", pp. 183-213 in Riemann surfaces and related topics (Stony Brook, NY, 1978), edited by I. Kra and B. Maskit, Ann. of Math. Stud. 97, Princeton Univ. Press, Princeton, N.J., 1981. MR 82m:53035 Zbl 0467.53035

[Ozawa and Rieffel 2003] N. Ozawa and M. A. Rieffel, "Hyperbolic group $C *$-algebras and freeproduct $C *$-algebras as compact quantum metric spaces", preprint, 2003. math.OA/0302310

[Rieffel 1998] M. A. Rieffel, "Metrics on states from actions of compact groups", Doc. Math. 3 (1998), 215-229. MR 99k:46126 Zbl 0993.46043

[Rieffel 1999] M. A. Rieffel, "Metrics on state spaces", Doc. Math. 4 (1999), 559-600. MR 2001g: 46154 Zbl 0945.46052

[Rieffel 2002] M. A. Rieffel, “Group $C^{*}$-algebras as compact quantum metric spaces”, Doc. Math. 7 (2002), 605-651. MR 2004k:22009 Zbl 1031.46082

[Rieffel 2004] M. A. Rieffel, "Compact quantum metric spaces”, pp. 315-330 in Operator algebras, quantization, and noncommutative geometry, edited by R. S. Doran and R. V. Kadison, Contemp. Math. 365, Amer. Math. Soc., Providence, RI, 2004. MR 2005h:46099 Zbl 02153061

[Webster and Winchester 2003] C. Webster and A. Winchester, "Busemann points of infinite graphs", preprint, 2003. math.OA/0309291

Received January 16, 2004.

CORRAN WEBSTER

DEPARTMENT OF MATHEMATICAL SCIENCES

UNIVERSITY OF NEVADA LAS VEGAS

LAS VEGAS, NV 89154

cwebster@unlv.nevada.edu

www.nevada.edu/ cwebster/

ADAM WINCHESTER

MATHEMATICS DEPARTMENT

UNIVERSITY OF CALIFORNIA, LOS ANGELES

LOS ANGELES, CA 90095-1555

lagwadam@math.ucla.edu 